Check for updates

Cite this: Nanoscale Adv., 2019, 1, 1207

\title{
Sensitive and selective colorimetric nitrite ion assay using silver nanoparticles easily synthesized and stabilized by AHNDMS and functionalized with $\mathrm{PABA} \dagger$
}

\author{
Mohammed Hassan Ibrahim, (DD ab Zhonghua Xue, ${ }^{\text {a }}$ Hassan Idris Abdu, ${ }^{a}$ \\ Mahgoub Ibrahim Shinger, ${ }^{C}$ Ahmed Mahmoud Idris, ${ }^{a}$ Murtada Mohamed Edris, ${ }^{a}$ \\ Duoliang Shan ${ }^{\mathrm{a}}$ and Xiaoquan Lu (D) *a
}

Nitrite ions $\left(\mathrm{NO}_{2}{ }^{-}\right)$, as one of the important inorganic anions, exhibit considerable effects towards the environment and human health. Moreover, over intake of this anion may cause dangerous diseases. Herein, we successfully fabricated silver nanoparticles (AgNPs) using 4-amino-5-hydroxynaphthalene-2, 7-disulphonic acid monosodium salt (AHNDMS) and functionalized them with $p$-aminobenzoic acid (PABA), and used the functionalised AgNPs as a sensitive and selective colorimetric sensor for nitrite ions. The structure of the as-prepared pure AgNPs was experimentally characterized by different characterizations methods, namely, UV-Vis, FT-IR, CV, DPVs, SEM, TEM, and XRD. Additionally, the nitrite ion sensitively and selectively changes the brownish yellow color of the dispersed AgNPs to pinkish red, indicating aggregation of AgNPs, with a detection limit of $0.016 \mathrm{ppm}(0.348 \mu \mathrm{M})$ and $0.0069 \mathrm{ppm}(0.149$ $\mu \mathrm{M})$ by the naked-eye and by UV-vis spectroscopy, respectively. The color change suggested that the aggregation of AgNPs was induced by nitrite-selective diazo-coupling. UV-vis spectra show the disappearance of the absorbance at $474 \mathrm{~nm}$ and appearance of a new peak at $532 \mathrm{~nm}$, presumably due to the conversion of AgNPs to silver ions. Moreover, the studies of interference in the proposed sensor confirm its selectivity in the presence of anions as well as cations. Furthermore, linearity was observed between the absorption and the concentration of nitrite ions. More importantly, the proposed sensor was practicably applied for the determination of nitrite in different water samples, such as distilled water, river water, and tap water.

rsc.li/nanoscale-advances

\section{Introduction}

Nitrite $\left(\mathrm{NO}_{2}{ }^{-}\right)$ion, as one of the important inorganic anions and a nitrogen-containing nutrient, has been extensively used along with $\mathrm{NO}_{3}{ }^{-}$and $\mathrm{NH}_{4}{ }^{+}$in fertilizers for the improvement of plant growth. ${ }^{1-5} \mathrm{NO}_{3}{ }^{-}$also exists in the environment systems, food products, and in the biological nitrogen cycle as a significant mediator. ${ }^{6}$ Additionally, $\mathrm{NO}_{2}{ }^{-}$is often used in meat and fish product preservation, and can interact with amines to produce nitrosamines, which have been distinguished as carcinogenic compounds, subsequently increasing the chances of cancer and malformation. ${ }^{7-10}$ Moreover, excess intake of $\mathrm{NO}_{2}{ }^{-}$will cause

${ }^{a}$ Key Laboratory of Bioelectrochemistry \& Analysis of Gansu Province, College of Chemistry \& Chemical Engineering, Northwest Normal University, Lanzhou, 730070, P. R. China. E-mail: xzhlab@hotmail.com; luxq@nwnu.edu.cn

${ }^{b}$ Department of Chemical Engineering, Faculty of Engineering and Technical Studies, University of Kordofan, El-Obeid, Sudan

${ }^{c}$ Department of Chemistry, International University of Africa, Khartoum, Sudan

$\dagger$ Electronic supplementary information (ESI) available: From Fig. S1 to S5. See DOI: $10.1039 / \mathrm{c} 8$ na00146d several medical problems, such as methemoglobinemia, which is commonly known as "blue baby syndrome", and esophageal cancer. ${ }^{11,12}$ The U.S. Environmental Protection Agency (EPA) recommended that the maximum limit for $\mathrm{NO}_{2}{ }^{-}$in drinking water should be $c a .21 .74 \mu \mathrm{M}$ or $1 \mathrm{ppm} .{ }^{13}$ Thus, the qualitative and quantitative analysis of $\mathrm{NO}_{2}{ }^{-}$in water, food and agricultural products is of vital importance.

Numerous analytical approaches, such as chromatography, ${ }^{14,15}$ spectroscopy, ${ }^{16,17}$ Raman spectroscopy, ${ }^{18,19}$ fluorescence, ${ }^{\mathbf{9} 20}$ flow injection system analysis, ${ }^{\mathbf{2 1}, 22}$ chemiluminescence analysis, ${ }^{23,24}$ and electrochemistry, ${ }^{6,25,26}$ have been developed for $\mathrm{NO}_{2}{ }^{-}$testing and monitoring, using which the nitrite ion sensitively and selectively can be detected. Unfortunately, the use of these techniques requires expensive and complicated instruments with highly trained operators that need experience in dealing with them. Therefore, their application in routine detection is limited. Recently, rapid detection methods for $\mathrm{NO}_{2}{ }^{-}$are being preferred, particularly for the visual on-site analysis because $\mathrm{NO}_{2}{ }^{-}$is known to be chemically unstable. ${ }^{5}$ 
Colorimetric sensors are particularly fascinating due to their low-cost, simplicity, sensitivity, and rapidity in analysis of ions. ${ }^{27-37} \mathrm{NO}_{2}{ }^{-}$optical sensors generally utilize selective diazocoupling reaction, in which $\mathrm{NO}_{2}{ }^{-}$can react with an aromatic amine in acidic medium to produce a diazonium salt that consequently couples with another aromatic compound to form a highly colored azo dye. ${ }^{38}$ Unluckily, there are some disadvantages: many methods based on this special reaction are not sensitive enough for $\mathrm{NO}_{2}{ }^{-}$at low concentrations, use hazardous reagents at a high concentration in the reaction and consume a lot of time for adjusting to a particular $\mathrm{pH}$ solution and temperature for the coupling reaction, hence resulting in a complicated method. However, developing high-performance sensing systems and highly stable, environmentally friendly, simple, facile and economical methods with superior properties still remain a great challenge for researchers. Very recently, silver and gold nanoparticles (NPs) have been found to be particularly appealing for fabricating and constructing colorimetric sensors due to their attractive properties, such as powerful surface plasmon resonance, and distance-dependent optical properties, as their performance events can be easily observed by the naked-eye as visible color changes. ${ }^{39-42}$ In this way, there is no need of complicated instruments and tedious detection methods. Moreover, NPs have been used successfully for the selective colorimetric detection of many species with excellent absorption coefficients and low detection limits. ${ }^{\mathbf{4 3 , 4 4}}$ Therefore, a variety of metal cation colorimetric sensing methods involving NPs have been widely explored. ${ }^{45-49}$

The sensing of anions, particularly $\mathrm{NO}_{2}{ }^{-}$, is rarely reported using noble NPs-based colorimetric sensors. Mirkin et al. ${ }^{\mathbf{5 0}}$ fabricated an AuNPs sensor for colorimetric $\mathrm{NO}_{2}{ }^{-}$detection, in which nitrite ion induced the aggregation of the AuNPs and caused a color change through the diazo-coupling reaction, with a detection limit $21.74 \mu \mathrm{M}$. However, this method included designing two types of functionalized AuNPs, which was time consuming. Xiao et al. ${ }^{51}$ designed 4-aminothiophenol gold nanorods (AuNRs) modified as a colorimetric $\mathrm{NO}_{2}{ }^{-}$sensor based on the reduction of the AuNRs surface charges, followed by aggregation of the AuNRs in the presence of nitrite ion, with detection limit below $21.74 \mu \mathrm{M}$. However, in this strategy, heating is necessary. Chen et al. ${ }^{52}$ developed a colorimetric method for $\mathrm{NO}_{2}{ }^{-}$detection based on the etching of AuNRs, the detection limit using this method is $4.0 \mu \mathrm{M}$. Heating is also needed here and moreover, there is interference from ferric ions $\left(\mathrm{Fe}^{3+}\right)$. Kumar et al. ${ }^{53}$ reported a colorimetric sensor based on AgNPs stabilized by the phenolic chelating ligand, in which the addition of the $\mathrm{NO}_{2}{ }^{-}$caused the decolorization of brownish yellow color of the as-synthesized AgNPs with a good detection limit of $0.1 \mu \mathrm{M}$. Yet, the addition process is needed here to synthesize the phenolic chelating ligand, which results in large time consumption. The disadvantages mentioned with the above systems may limit their application in routine real sample analysis.

Motivated by the appealing properties of the NPs, in this study, we designed a facile and improved system based on AgNPs, which were synthesized and stabilized by AHNDMS and functionalized with PABA for colorimetric $\mathrm{NO}_{2}{ }^{-}$sensing for the first time. Herein, the addition of nitrite ions to the asprepared AgNPs in alkaline media results in a color change from brownish yellow to pinkish red, which is suggested to indicate the $\mathrm{NO}_{2}{ }^{-}$-induced aggregation of the AgNPs by selective diazo-coupling strategy without heat treatment. There is also no need to synthesize two types of functionalized NPs, and no additional processes are required to prepare functionalization agents. The detection limit by the naked-eye is $0.016 \mathrm{ppm}(0.348 \mu \mathrm{M})$ and $0.0069 \mathrm{ppm}(0.149 \mu \mathrm{M})$ by UV-vis spectroscopy, with no interferences observed from the presence of other ions. Thus, this present system is simpler and more sensitive and selective than the formerly reported methods. On top of this, we have introduced a multifunctional compound (AHNDMS) as a novel reducing and stabilizing agent, which could electrostatically interact with silver ions through $-\mathrm{OH},{ }^{45,54},-\mathrm{NH}_{2},{ }^{55}$ and $-\mathrm{S}=\mathrm{O}^{56}$ groups to form dispersed AgNPs.

\section{Experimental section}

\subsection{Reagents}

AHNDMS was obtained from Aladdin. A stock sodium nitrite solution with concentration of $1000 \mu \mathrm{g} \mathrm{mL} \mathrm{m}^{-1}$ was purchased from Guobiao (Beijing) Testing \& Certification Co., Ltd. PABA was purchased from Sinopharm Chemical Reagent Co., Ltd. All other chemicals used were of analytical grade and their solutions were prepared using double distilled water $(>18.25 \mathrm{M} \Omega$ $\mathrm{cm})$. Working solutions were prepared by diluting the standard solution of nitrite ion to an appropriate volume with double distilled water, whenever required. All experiments were performed at room temperature.

\subsection{Synthesis and functionalization of AgNPs}

AgNPs were synthesized using a modified previously reported method. ${ }^{53}$ In addition, AHNDMS, which we used for the first time as a new reducing-capping agent, easily converted silver ions to silver nanoparticles. Silver nitrate solution $(500 \mu \mathrm{M})$ was added into an aqueous solution of AHNDMS $(500 \mu \mathrm{M})$, and the total volume of the final reaction solution was $40 \mathrm{~mL}$. The contents were stirred vigorously at $70{ }^{\circ} \mathrm{C}$. After a few minutes, brownish yellow color appeared, suggesting the reduction of silver ions into AgNPs. The solution was allowed to stir at the same conditions for more than $2 \mathrm{~h}$ and for $1 \mathrm{~h}$ at room temperature. The synthesis procedure was repeated several times to verify and confirm the reproducibility of the AgNPs. Characterization, functionalization, and other measurements of the obtained AgNPs were performed after allowing the solution to stand at room temperature for $24 \mathrm{~h}$. Functionalization of the as-synthesized AgNPs with PABA was performed by the addition of acidified PABA solution to an appropriate amount of the as-prepared AgNPs, such that the final concentration of PABA in the reaction container was $7 \mathrm{mM}$, followed by strong stirring for $2 \mathrm{~h}$ at room temperature. 


\subsection{Recommended procedure}

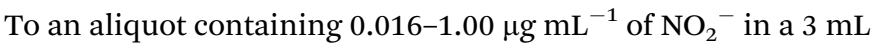
calibration tube, $0.5 \mathrm{~mL}$ of the AgNPs solution and $0.5 \mathrm{~mL}$ of buffer solution ( $\mathrm{pH}=9.4$ ) were added, upon which the color of the solution immediately changed to pinkish red. The contents of each tube were diluted to the mark with double distilled water, shaken thoroughly, and kept aside for $35 \mathrm{~min}$ at room temperature before the absorbance of the pinkish red colored solution was measured at $532 \mathrm{~nm}$ against reagent blank. Beer's law graph was constructed by plotting absorbance against $\mathrm{NO}_{2}{ }^{-}$ concentrations.

\subsection{Selectivity of proposed sensor}

To verify the selectivity of the proposed detection system, other ions (anions and cations), namely, nitrate $\left(\mathrm{NO}_{3}{ }^{-}\right)$, chloride $\left(\mathrm{Cl}^{-}\right)$, fluoride $\left(\mathrm{F}^{-}\right)$, thiocyanide $\left(\mathrm{SCN}^{-}\right)$, carbonate $\left(\mathrm{CO}_{3}{ }^{-}\right)$, bicarbonate $\left(\mathrm{HCO}_{3}{ }^{-}\right)$, oxalate $\left(\mathrm{C}_{2} \mathrm{O}_{4}{ }^{2-}\right)$, sulphate $\left(\mathrm{SO}_{4}{ }^{2-}\right)$, dihydrogen phosphate $\left(\mathrm{H}_{2} \mathrm{PO}_{4}{ }^{-}\right)$, hydrogen phosphate $\left(\mathrm{HPO}_{4}{ }^{2-}\right)$, manganese $\left(\mathrm{Mn}^{2+}\right)$, iron(II) $\left(\mathrm{Fe}^{2+}\right)$, iron(III) $\left(\mathrm{Fe}^{3+}\right)$, cobalt $\left(\mathrm{Co}^{2+}\right)$, nickel $\left(\mathrm{Ni}^{2+}\right)$, copper $\left(\mathrm{Cu}^{2+}\right)$, zinc $\left(\mathrm{Zn}^{2+}\right)$, calcium $\left(\mathrm{Ca}^{2+}\right)$, magnesium $\left(\mathrm{Mg}^{2+}\right)$, and lead $\left(\mathrm{Pb}^{2+}\right)$, were tested with the recommended method. The concentration of other ions $(1000 \mu \mathrm{M})$ used in this investigation was 50-fold higher than that of $\mathrm{NO}_{2}{ }^{-}$, except that of $\mathrm{Mn}^{2+}, \mathrm{Fe}^{2+}$, and $\mathrm{Fe}^{3+}$, which were $200 \mu \mathrm{M}$ (10-fold higher than that of $\mathrm{NO}_{2}{ }^{-}$).

\subsection{Real samples analysis}

Different water samples such as deionized water, yellow river water, and tap water were investigated using the recommended method after spiking with $\mathrm{NO}_{2}{ }^{-}$at different concentrations for the measurement of recovery. Yellow river (China-Lanzhou) water sample was collected in a polyethylene container, filtered and assayed directly. Deionized water and tap water samples were collected from the lab at Northwest Normal University in Lanzhou, and immediately analyzed without any additional treatment.

\subsection{Characterization}

UV-vis experiments were performed using an Agilent UV-8453 spectrophotometer (Agilent Inc., Japan). The Fourier transform infrared (FTIR) spectra were recorded on a Bruker Vertex 70 FT-IR spectrophotometer using the KBr method. Electrochemical measurements were performed on a CHI660 electrochemical station (CHI Instruments Inc., USA) with a conventional three-electrode system, where bare and/or modified glassy carbon electrodes (GCE, $3 \mathrm{~mm}$ in diameter) were used as the working electrodes and Pt wire and $\mathrm{Ag} / \mathrm{AgCl}$ (saturated $\mathrm{KCl}$ ) were used as the auxiliary and reference electrodes, respectively. Transmission electron micrographs were taken using a FEI Teena G2 F20 transmission electron microscope (TEM). X-ray diffraction (XRD) was detected on an X-ray diffractometer (Bruker AXS). A dried powder of AgNPs was used for XRD measurements. Scanning electron microscopy (SEM) was conducted using a Zeiss electron microscope (Zeiss, Oberkochen, Germany).

\section{Results and discussion}

Phenolic compounds have the ability to reduce silver ions and stabilize AgNPs through electrostatic interactions of the phenolic oxygen atoms. ${ }^{53,54}$ It was reported that bifunctional compounds, such as melamine ${ }^{47,55}$ and Gallic acid, ${ }^{57}$ can reduce silver ions to AgNPs. Hence, mixing of $\mathrm{AgNO}_{3}$ solution with AHNDMS solution of certain concentration followed by vigorous stirring under $70{ }^{\circ} \mathrm{C}$ produced a brownish yellow solution of monodispersed AgNPs with maximum absorption at $474 \mathrm{~nm}$, which may be due to the surface plasmon resonance (SPR) vibration of silver. ${ }^{58}$ Addition of PABA solution to the AgNPs at certain amounts followed by stirring retained the solution color (brownish yellow) with the same absorption peak of the pure formed AgNPs, indicating no aggregation of AgNPs. In the presence of $\mathrm{NO}_{2}{ }^{-}$under acidic conditions, the amine group of the PABA or AHNDMS converted to a diazonium salt, which then coupled with the aromatic group on the same compounds to form an azo group in alkaline medium. Subsequently, aggregation of the AgNPs took place, shortening the interparticle distance, which caused a drastic color change from brownish yellow to pinkish red, as illustrated in Scheme 1.

In order to support and confirm the mechanism suggested above, UV-visible investigations were performed and depicted in Fig. 1. The pure AgNPs prepared by AHNDMS show an absorption peak at $474 \mathrm{~nm}$, which agrees well with AgNPs SPR (curve (a) and inset image (a)). Curve (b) and inset image (b) show that the addition of PABA for functionalization cannot change the absorbance peak position (434 nm) and brownish yellow solution color; instead, it just caused a small decrease in the absorption value, suggesting an interaction between AgNPs and $\mathrm{PABA}$ via $-\mathrm{COOH}$, and $-\mathrm{NH}_{2}$ groups, as reported earlier. ${ }^{\mathbf{5 9 , 6 0}}$ On mixing $0.5 \mathrm{~mL}$ of the as-formed AgNPs with $0.5 \mathrm{~mL}$ buffer solution $(\mathrm{pH}=9.4)$ in a $3 \mathrm{~mL}$ calibration tube followed by diluting the solution with double distilled water to the mark, the absorption peak decreased sharply, while its position remained at $474 \mathrm{~nm}$, and the color of the solution changed to pale-yellow due to the dilution of the AgNPs solution (curve (c) and inset image (c)). Addition of the $\mathrm{NO}_{2}{ }^{-}$solution of a certain concentration to $0.5 \mathrm{~mL}$ of AgNPs was studied in the same manner as explained in the recommended procedure. A new

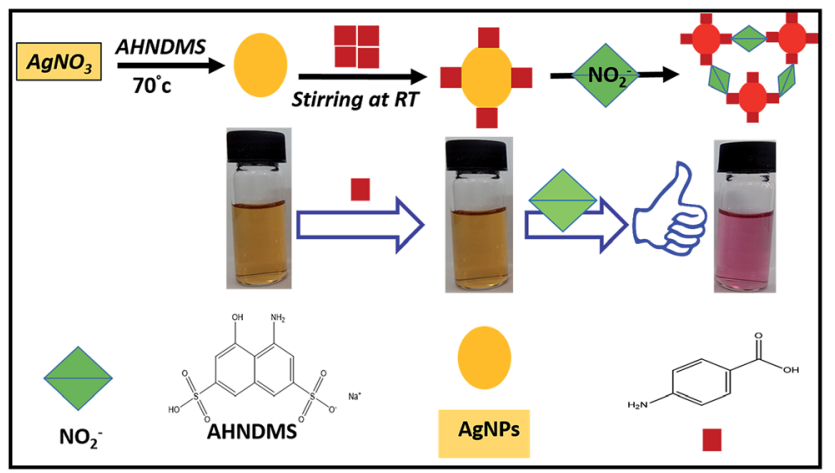

Scheme 1 Schematic for colorimetric sensing of $\mathrm{NO}_{2}^{-}$using AgNPs synthesized by AHNDMS and functionalized with PABA. 


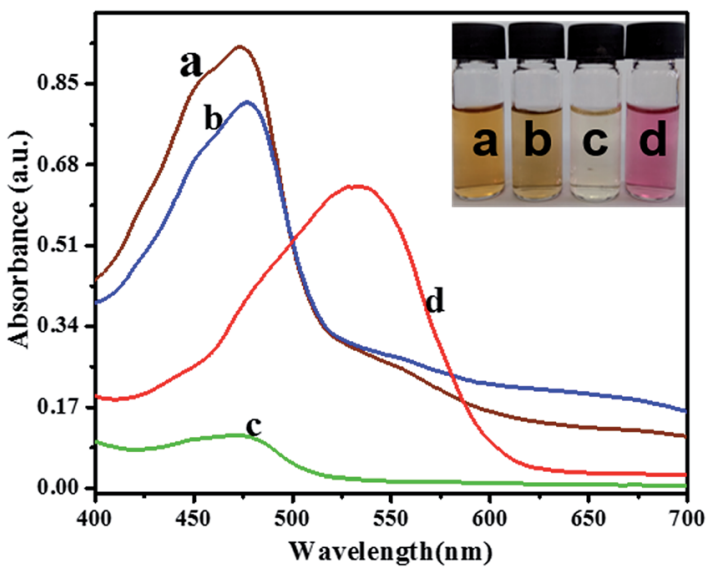

Fig. 1 Absorption spectra of the AgNPs (a), functionalized AgNPs (b), $0.5 \mathrm{~mL}$ (b) $+0.5 \mathrm{~mL} \mathrm{pH}=9.4$ buffer solution $+2 \mathrm{~mL} \mathrm{DW}$ (c), and (c) $+1 \mathrm{ppm} \mathrm{NO}_{2}^{-}$(d). Inset is the photograph of corresponding solutions.

absorption peak appeared at $532 \mathrm{~nm}$ (curve (b)), corresponding to the pinkish red color change for the solution (inset image (b)). This response is attributed to the AgNPs aggregation induced by $\mathrm{NO}_{2}{ }^{-}$due to the reasons discussed above and reported previously, ${ }^{50}$ and was further confirmed by SEM and TEM in Fig. 4 and by CV and DPVs in Fig. S2. $\dagger$

The infrared spectra of the pure AHNDMS (curve (a)), pure PABA (curve (b)), as-prepared AgNPs (curve (c)), and functionalized AgNPs (curve (d)) are comparatively assembled and depicted in Fig. 2, where characteristic features are observed. After reaction of AHNDMS with $\mathrm{AgNO}_{3}$, the peaks shifted to either higher or lower wave number. The peaks at $870 \mathrm{~cm}^{-1}(\mathrm{~N}-$ $\mathrm{H}$ bending), $1148 \mathrm{~cm}^{-1}$ ( $\mathrm{S}=\mathrm{O}$ stretching), $1372 \mathrm{~cm}^{-1}$ (C-N stretching), $1501 \mathrm{~cm}^{-1}$ (N-H bending) $3396 \mathrm{~cm}^{-1}(\mathrm{~N}-\mathrm{H}$ stretching), and $3527 \mathrm{~cm}^{-1}$ (O-H stretching) in the AHNDMS spectrum shifted to $888 \mathrm{~cm}^{-1}, 1187 \mathrm{~cm}^{-1}, 1400 \mathrm{~cm}^{-1}$, $1530 \mathrm{~cm}^{-1}, 3432 \mathrm{~cm}^{-1}$ and $3784 \mathrm{~cm}^{-1}$, respectively in the AgNPs spectrum. After introducing PABA to the AgNPs solution, IR spectrum of the functionalized AgNPs shows peaks at $742 \mathrm{~cm}^{-1}$,

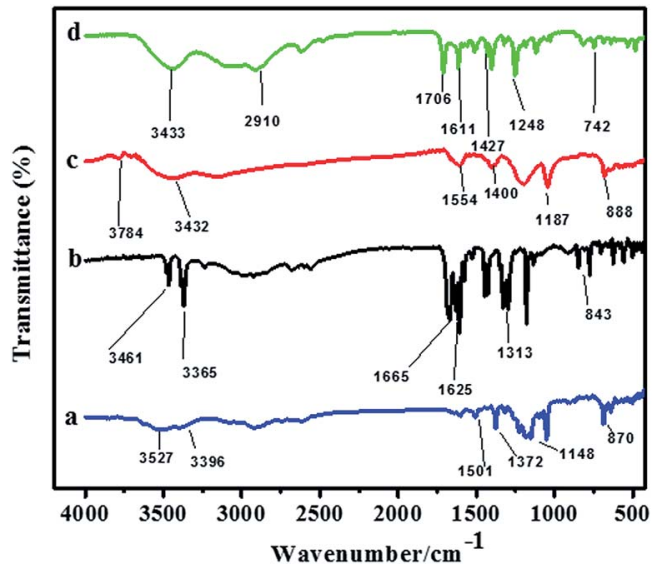

Fig. 2 FT-IR spectra of AHNDMS (a), PABA (b), synthesized AgNPs (c), and functionalized AgNPs (d).
$1174 \mathrm{~cm}^{-1}, 1427 \mathrm{~cm}^{-1}, 1554 \mathrm{~cm}^{-1}, 3011 \mathrm{~cm}^{-1}$ and $3433 \mathrm{~cm}^{-1}$ due to the shifting of $843 \mathrm{~cm}^{-1}$ (aromatic $\mathrm{N}-\mathrm{H}$ bending), $1313 \mathrm{~cm}^{-1}$ (C-N stretching), $1573 \mathrm{~cm}^{-1}$ (N-H bending), $1625 \mathrm{~cm}^{-1}$ (aromatic rings), $1665 \mathrm{~cm}^{-1}(\mathrm{C}=\mathrm{O}$ stretching), $3365 \mathrm{~cm}^{-1}$ ( $\mathrm{N}-\mathrm{H}$ stretching), and $3461 \mathrm{~cm}^{-1}$ (O-H stretching) peaks of the PABA spectrum. All the above peak shifts were due to the binding of $\mathrm{N}-\mathrm{H}, \mathrm{S}=\mathrm{O}, \mathrm{C}-\mathrm{N}, \mathrm{N}-\mathrm{H}$, and $\mathrm{O}-\mathrm{H}$ groups of PABA with the as-formed AgNPs, indicating the successful reduction of $\mathrm{Ag}^{+}$to AgNPs, capping with AHNDMS, and subsequent functionalization with PABA through $-\mathrm{NH}_{2}$ group and - $\mathrm{COOH}$ group of $p$-aminobenzoic acid. To clarify the sensing mechanism of $\mathrm{NO}_{2}{ }^{-}$, the IR spectra of the as-prepared AgNPs, AgNPs functionalized with PABA (AgNPs/PABA), and AgNPs/ PABA in the presence of nitrite ions in alkaline medium $\left(\mathrm{AgNPs} / \mathrm{PABA}+\mathrm{NO}_{2}{ }^{-}\right.$) were collected, as shown in Fig. S1. $\dagger$ The IR spectra of the as-prepared pure AgNPs and AgNPs/PABA are already revealed in Fig. 2. The AgNPs/PABA $+\mathrm{NO}_{2}{ }^{-}$spectrum shows new peaks at $1143 \mathrm{~cm}^{-1}, 1395 \mathrm{~cm}^{-1}$ and $1456 \mathrm{~cm}^{-1}$, corresponding to the $\mathrm{N}=\mathrm{N}$ bond stretching, ${ }^{1,61}$ indicating that the nitrite ions have undergone diazo-coupling reaction, as we have suggested previously.

The crystalline phase and the structural purity of AgNPs were investigated by XRD technique. The distinct and characteristics peaks obtained in the XRD pattern of AgNPs (Fig. 3) further confirmed the existence of the nanocrystalline structure of AgNPs. The Bragg reflection peaks at $2 \theta$ values of $27.849^{\circ}$, $32.278^{\circ}, 38.149^{\circ}, 44.372^{\circ}, 46.245^{\circ}, 54.770^{\circ}, 57.481^{\circ}, 64.387^{\circ}$, and $77.418^{\circ}$ were assigned to the corresponding (210), (122), (111), (231), (200), (142), (241), (220), and (311) planes for AgNPs based on the face-centered cubic structure (JCPDS file no. 040783). This result suggests that the as-formed AgNPs are biphasic and crystalline in nature. XRD analysis results are displayed in Fig. S2 $\uparrow$ to support the sensing mechanism of the sensor; the XRD pattern of AgNPs (green curve) is already revealed in Fig. 3. The XRD pattern of AgNPs/PABA (blue curve) represents the functionalized AgNPs pattern, which contains majority of the AgNPs peaks besides the undefined peaks due to the presence of PABA, indicating that the AgNPs have been 
successfully functionalized with PABA. The XRD pattern of AgNPs/PABA $+\mathrm{NO}_{2}{ }^{-}$(red curve) shows how the existence of nitrite ions in the sensor changes the crystalline phase of AgNPs/PABA and produces a new XRD pattern for silver. The red curve shows peaks at $2 \theta$ values of $27.849^{\circ}, 32.278^{\circ}, 38.149^{\circ}$, $46.245^{\circ}, 57.481^{\circ}, 68.387^{\circ}$, and $77.418^{\circ}$, corresponding to the (210), (122), (111), (200), (142), (220), and (311) planes for silver. $^{62}$ However, these peaks in comparison with those of AgNPs are different in terms of intensity values, with shifts occurring for many of them. This investigation confirmed the formation of silver ions, which occurred by $\mathrm{NO}_{2}{ }^{-}$inducing the aggregation of AgNPs by a diazo-coupling reaction. These results agree well with the results reported in the UV-visible investigation and with the mechanism suggested in Scheme 1. The XRD measurements for AgNPs characterization are in good agreement with the previous reports. ${ }^{63-67}$

For nanostructural studies, surface morphology of the obtained AgNPs was characterized by using Scanning Electron Microscopy (SEM). The results are summarized and depicted in Fig. 4. As shown in the figure, the AgNPs have a spherical shape (Fig. 4A). No significant change is observed in the shape of the AgNPs even after functionalization with PABA (Fig. 4B). Interestingly, when nitrite ions were introduced at a certain concentration to the functionalized AgNPs under the optimum conditions, the spherical AgNPs changed to aggregated particles (Fig. 4C), indicating that the presence of $\mathrm{NO}_{2}{ }^{-}$in the sensor induced the aggregation of the functionalized AgNPs by selective diazotization coupling reactions in the nanoparticle system.

For further confirmation of the sensing mechanism, the assynthesized AgNPs were characterized using Transmission Electron Microscopy technique (TEM). TEM revealed that the AgNPs formed by AHNDMS were predominately oval and spherical (Fig. 4D). There is no change in the nanoparticles even after treatment with PABA as the functionalization compound (Fig. 4E). Significant aggregation of AgNPs was observed when $\mathrm{NO}_{2}{ }^{-}$was introduced into the senor system under optimum
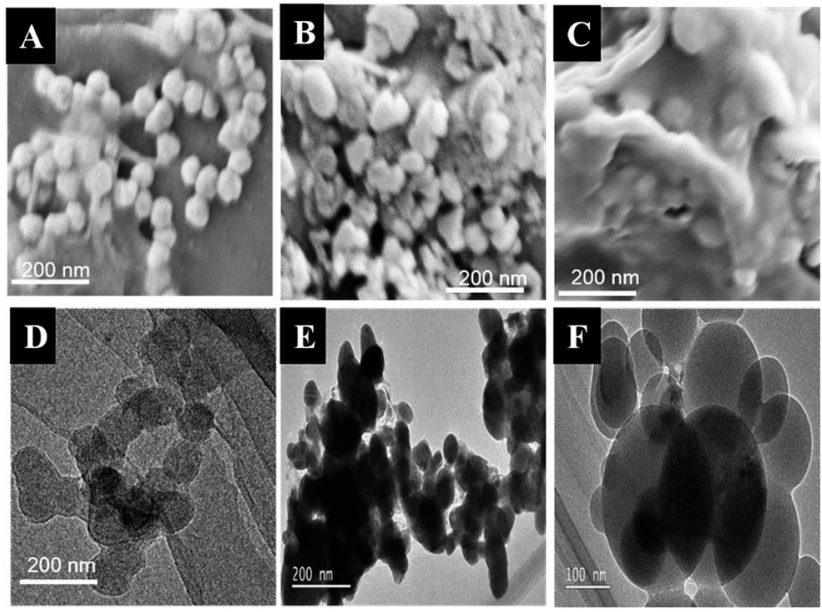

Fig. 4 SEM images of the as-synthesized AgNPs (A), functionalized AgNPs (B), and aggregated AgNPs (C). TEM images of the synthesized AgNPs (D), functionalized AgNPs (E), and aggregated AgNPs (F). conditions, as shown in Fig. 4F. The as-synthesized AgNPs form regular spheres with an average diameter of $53.85 \mathrm{~nm}$ (Fig. S3A $\dagger$ ). After functionalization with PABA (Fig. S3B $\dagger$ ) and introduction of $\mathrm{NO}_{2}{ }^{-}$to the sensor system (Fig. S3C $\dagger$ ), the average size increased to $57.62 \mathrm{~nm}$ and $132.83 \mathrm{~nm}$, respectively. All these investigations confirmed the formation of AgNPs, the subsequent functionalizing with PABA, and the aggregation by $\mathrm{NO}_{2}{ }^{-}$through the mechanism under discussion. The SEM and TEM results agree well with the suggestion in Scheme 1 and the UV-vis spectroscopy indicating color change in Fig. 1.

Cyclic voltammetry (CV) investigations of the proposed AgNPs were achieved and depicted in Fig. S4A. $\dagger$ The CV response of pure AHNDMS solution in $\mathrm{pH} 9.4$ (curve (a)) shows a clear anodic peak at $0.315 \mathrm{~V}$. The reaction solution of AHNDMS with $\mathrm{AgNO}_{3}$ at $\mathrm{pH} 9.4$ (curve (b)) exhibits a CV response with two anodic and cathodic peaks at $0.365 \mathrm{~V}$ and $-0.103 \mathrm{~V}$, respectively, which may be due to the formation of AgNPs by AHNDMS, corresponding to the change from colorless $\mathrm{AgNO}_{3}$ to a yellow brownish solution in Fig. 1. CV response of AgNPs/PABA at $\mathrm{pH}=9.4$ (curve (c)) reveals a distinct cathodic peak at $0.048 \mathrm{~V}$, possibly because of the functionalization of capped AgNPs with PABA by ligand exchange interaction. On introducing $\mathrm{NO}_{2}{ }^{-}$to the proposed sensor at $\mathrm{pH} 9.4$, the color of the solution changed to pinkish red, and its $\mathrm{CV}$ response (curve (d)) shows two peaks, viz., anodic and cathodic at $0.820 \mathrm{~V}$ and $-0.42 \mathrm{~V}$, respectively, which are expected to be due to the aggregation of AgNPs by the same reasons we have suggested before, corresponding to the solution color change from brownish yellow to pinkish red in Fig. 1 and agreeing with the suggested mechanism in Scheme 1.

A Differential Pulse Voltammetry measurement was performed for further confirmation of the proposed sensor. As illustrated in Fig. S4B, $\uparrow$ the curve of pure AHNDMS exhibits no visible current-sensitive peaks, indicating a shortage of species for a redox reaction (curve (a)). After reduction of $\mathrm{AgNO}_{3}$ to AgNPs by AHNDMS (curve (b)), five current-sensitive wavy peaks appeared due to an increase in the species by redox reaction, which corresponds to the change of the colorless $\mathrm{AgNO}_{3}$ solution to the brownish yellow color of AgNPs, as shown in Fig. 1. These five wave currents drastically increased after AgNPs were functionalized with PABA (curve (c)). Moreover, two of these peaks disappeared, while another two remained without a change in current, and the remaining peak's current increased drastically, which indicates the aggregation of AgNPs when the target $\mathrm{NO}_{2}{ }^{-}$ion was introduced to the sensor (curve (d)), which corresponded to the color change from brownish yellow to pinkish red in Fig. 1. This result also supports the mechanism we suggested in Scheme 1.

Effects of different conditions leading to inappreciable sensing and detection of nitrite ions by the proposed colorimetric sensor were investigated. As displayed in Fig. S5, $\uparrow$ in the $\mathrm{pH}$ range of 7.2 to $13,0.5 \mathrm{~mL}$ volume of buffer solution was added to a series of fixed nitrite concentrations in the sensor, with final volume of $3 \mathrm{~mL}$ in the calibrated tubes. The maximum absorption with good linearity was obtained at $\mathrm{pH}$ 9.4 when the absorption at $532 \mathrm{~nm}$ was plotted against the $\mathrm{pH}$ value in Fig. S5A. $\uparrow$ Variation in the absorption value of color was 
observed between 2 min and 45 min with the addition of buffer solution to a series of fixed nitrite amounts and $0.5 \mathrm{~mL}$ of synthesized AgNPs in a $3 \mathrm{~mL}$ volumetric tube. The maximum absorption value at $532 \mathrm{~nm}$ with good linearity was observed after 35 minutes, as shown in Fig. S5B. $\uparrow$ The concentration effect of PABA functionalization with AgNPs on the absorption value was studied by changing the PABA concentrations from $1.5 \mathrm{mM}$ to $22 \mathrm{mM}$, as shown in Fig. S5C, $\dagger$ by plotting absorption values at $532 \mathrm{~nm}$ against PABA concentrations. It was demonstrated that the maximum absorption with good linearity was with $7 \mathrm{mM}$ of PABA. Finally, the effect of hydrochloric acid used for dissolving PABA for functionalization of AgNPs was studied by using different concentrations of hydrochloric acid ranging from $16 \mathrm{mM}$ to $160 \mathrm{mM}$; it was observed that the maximum absorption at $532 \mathrm{~nm}$ with good linearity was with $80 \mathrm{mM} \mathrm{HCl}$ (Fig. S5D †).

The selective colorimetric investigation of the proposed method towards $\mathrm{NO}_{2}{ }^{-}\left(1 \mathrm{ppm}\right.$ equal $\left.2.17 \times 10^{-5} \mathrm{M}\right)$ was studied by the addition of metal and other ions (anions and cations) at concentrations of $1000 \mu \mathrm{M}$ (except for manganese $\left(\mathrm{Mn}^{2+}\right)$, iron(II) $\left(\mathrm{Fe}^{2+}\right)$, and iron(III) $\left(\mathrm{Fe}^{3+}\right)$, which were at $\left.200 \mu \mathrm{M}\right)$ to the detection solution under optimized conditions. As seen in Fig. 5A, for anions after their addition to the sensing solution, no change was observed in the color of the sensing solution, while there was a distinct change from brownish yellow to pinkish red observed after addition of $\mathrm{NO}_{2}{ }^{-}$. Additionally, no change in the intensity of the peak of the precursor AgNPs in the presence of other anions was observed, while the addition of nitrite ion resulted in the appearance of a new peak with maximum absorption value at $532 \mathrm{~nm}$ (Fig. 5B). These color changes are confirmed in Fig. 5C as a histogram curve by plotting the absorption values at $532 \mathrm{~nm}$ against the anions added. Moreover, when some cation additions were investigated, no visible color changes (Fig. 6A), no shifts in the position of the original peak of AgNPs (Fig. 6B) and no significant changes in the absorption (Fig. 6C) were observed with any ions except with nitrite ions. For the ions with concentration of 1000 $\mu \mathrm{M}$ and higher, there is no change during sensing with the proposed sensor, and the color remains clear brownish yellow.

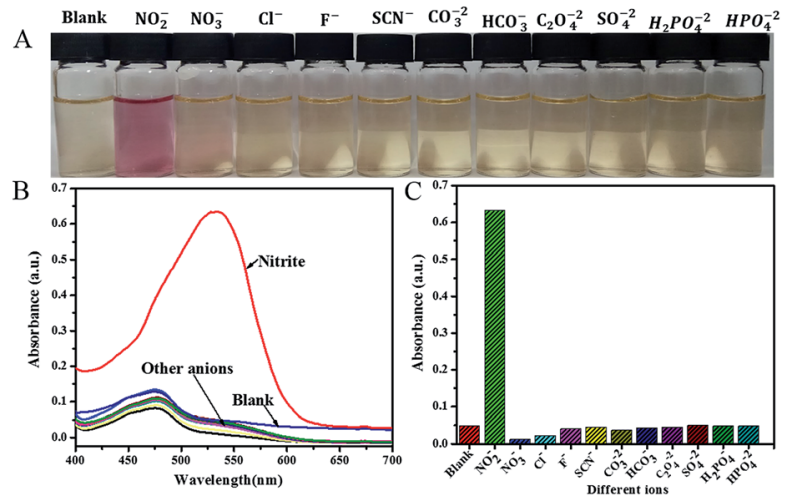

Fig. 5 (A) The corresponding color changes of (B) UV-vis absorption spectra of the sensing of different anions and nitrite $\left(\mathrm{NO}_{2}{ }^{-}\right)$ions by the recommended procedure. (C) Absorption enhancement of the anions and nitrite at $532 \mathrm{~nm}$.
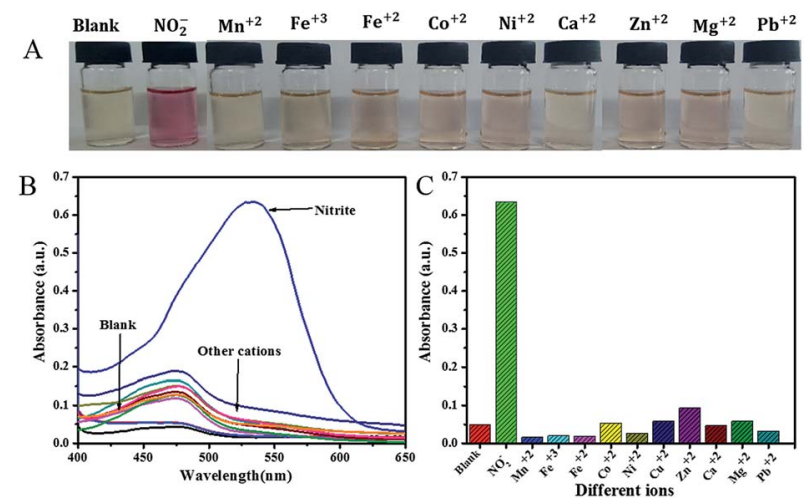

Fig. 6 (A) The corresponding color change of (B) UV-vis absorption spectra of the sensing of different cations and nitrite $\left(\mathrm{NO}_{2}{ }^{-}\right)$by the recommended procedure. (C) Absorption enhancement of the cations and nitrite at $532 \mathrm{~nm}$.

However, on addition of ions $\mathrm{Mn}^{2+}, \mathrm{Fe}^{2+}$, and $\mathrm{Fe}^{3+}$ at concentrations more than $200 \mu \mathrm{M}$ to the sensing solution, the color intensity of the sensing solution increased. These results indicate that our proposed sensor has good selectivity for nitrite ions among many common ions.

UV-vis absorption spectrum of the functionalized AgNPs in the presence of nitrite ions with different concentrations under the optimized conditions was recorded. The proposed sensor displayed a good analytical performance for nitrite analysis. The color changes of the present system in the absence and the presence of varying amounts of nitrite ions were carefully studied. As depicted in Fig. 7A, a distinct color change from dark to light pinkish red color with the increase in nitrite ion concentration from 0 to $1 \mathrm{ppm}$ is observed. More importantly, the obtained optical change can be sensitively visualized by the naked-eye and is sufficient for analysis purpose. Fig. 7B illustrates that the absorbance at $532 \mathrm{~nm}$ gradually increased with the increase in concentration of the nitrite ions. The absorption values exhibited a good linear correlation with the concentration of nitrite ions in the range of 0.016-1 ppm (Fig. 7C). The regression equation is $A=0.59733(\mathrm{ppm})+0.04715$ with

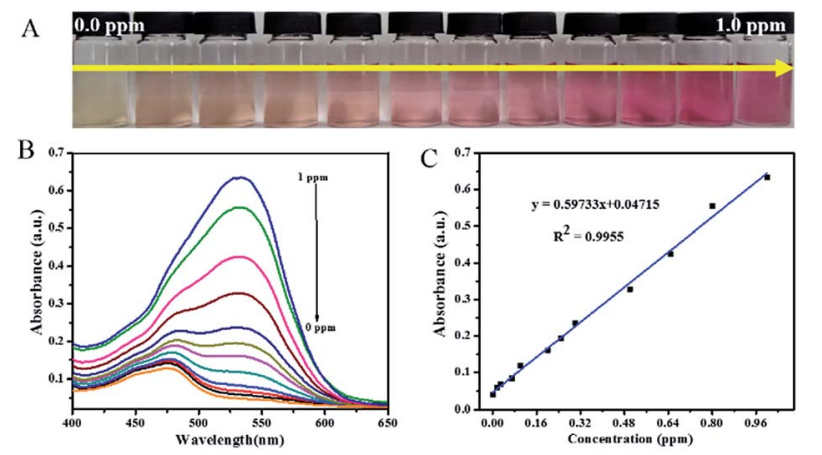

Fig. 7 (A) The corresponding solution colors of (B) UV-vis absorption spectra for the proposed sensing system with different concentrations of nitrite, and (C) the calibration response for the determination of nitrite. Concentrations range from 0.016 to $1 \mathrm{ppm}$. 
Table 1 Recoveries of the spiked nitrite ions in different water samples. The standard deviation of each sample was obtained from three measurements

\begin{tabular}{|c|c|c|c|c|}
\hline \multirow[t]{2}{*}{ Deionized water } & 0.5 & 0.46 & 92.30 & 0.74 \\
\hline & 1 & 0.96 & 95.70 & 0.14 \\
\hline \multirow[t]{3}{*}{ Tap water } & 0.5 & 0.41 & 82.67 & 1.14 \\
\hline & 1 & 0.91 & 90.50 & 0.32 \\
\hline & 2 & 1.88 & 99.50 & 3.26 \\
\hline & 2 & 1.87 & 93.63 & 0.22 \\
\hline
\end{tabular}

a correlation coefficient of 0.9955 . The detection limit of nitrite ion was calculated to be $0.0069 \mathrm{ppm}$ or $0.149 \mu \mathrm{M}$ (three measurements for each concentration) by UV-vis spectroscopy and $0.016 \mathrm{ppm}$ or $0.348 \mu \mathrm{M}$ by the naked-eye. Such performance characteristics suggested the potential use of the as-proposed colorimetric method for nitrite ion sensing.

In order to evaluate the appropriateness and unique properties of the proposed method, including rapid response, good selectivity, and sensitivity for nitrite ions, it was applied for the detection of nitrite ions in deionized water, tap water, and yellow river water. Different known amounts of nitrite ion solutions were directly spiked into the three real samples. The analyses results are summarized in Table 1. The average recoveries of the nitrite ion were in the range of $82.67-104.05 \%$ for all spiked samples with low relative standard deviations $(0.13-3.26 \%)$. This result indicates that such a method is applicable to the quantification of nitrite ions in various aqueous solutions.

\section{Conclusions}

In summary, a simple and rapid colorimetric sensor, with good selectivity and sensitivity, based on aggregation of AgNPs was synthesized and stabilized by AHNDSM for the first time. Following functionalization with PABA, the aggregation of brownish yellow AgNPs caused the appearance of pinkish red color with a maximum absorption at $532 \mathrm{~nm}$. The color change happened only when a specific concentration of the nitrite ions was added into the AgNPs solution at optimized conditions. The proposed method has been successfully applied for the colorimetric determination of nitrite ions, and it has been shown to be potentially useful for nitrite ion detection in real aqueous samples. The reagents are cheap and easily available. The developed color is stable for several days.

\section{Conflicts of interest}

There are no conflicts to declare.

\section{Acknowledgements}

We gratefully acknowledge the financial support from National Natural Science Foundation of China (Grants: 21665023,
21575115, 21327005, 21565021) and program for Chang Jiang Scholars, Ministry of Education, China (Grant No. IRT-16R61, 2017D-01) the Program of Innovation and Entrepreneurial for Talent, Lanzhou, Gansu province, China (Grant No. 2014RC-39).

\section{Notes and references}

1 Y. Xiong, M. Li, H. Liu, Z. Xuan, J. Yang and D. Liu, Nanoscale, 2017, 9, 1811-1815.

2 V. J. Sieben, C. F. Floquet, I. R. Ogilvie, M. C. Mowlem and H. Morgan, Anal. Methods, 2010, 2, 484-491.

3 J. A. Camargo and Á. Alonso, Environ. Int., 2006, 32, 831-849.

4 R. Michalski and I. Kurzyca, Pol. J. Environ. Stud., 2006, 15, 1230-1485.

5 A. Hatamie, M. Nassiri, M. D. Alivand and A. Bhatnagar, Talanta, 2018, 176, 156-164.

6 Z. Yilong, Z. Dean and L. Daoliang, Int. J. Electrochem. Sci., 2015, 10, 1144-1168.

7 S. M. Mali, P. P. Chavan, Y. H. Navale, V. B. Patil and B. R. Sathe, RSC Adv., 2018, 8, 11177-11185.

8 Y. Ye, Y. Guo, Y. Yue and Y. Zhang, Anal. Methods, 2015, 7, 4090-4096.

9 Y.-H. Zhan, R. Sun, W.-J. Zhu, Y.-J. Xu and J.-F. Ge, Sens. Actuators, B, 2017, 240, 1283-1290.

10 M. F. Salama, A. Abbas, M. M. Darweish, A. A. El-Hawwary and M. M. Al-Gayyar, Pharm. Biol., 2013, 51, 1435-1443.

11 T. A. Stueckle, D. C. Davidson, R. Derk, P. Wang, S. Friend, D. Schwegler-Berry, P. Zheng, N. Wu, V. Castranova and Y. Rojanasakul, Nanotoxicology, 2017, 11, 613-624.

12 H. Zhang, S. Qi, Y. Dong, X. Chen, Y. Xu, Y. Ma and X. Chen, Food Chem., 2014, 151, 429-434.

13 H. Yang and H. Cheng, Sep. Purif. Technol., 2007, 56, 392396.

14 M. Aristoy, M. Reig and F. Toldrá, Adv. Food Diagn., 2017, 229-251.

15 S. Chamandust, M. R. Mehrasebi, K. Kamali, R. Solgi, J. Taran, F. Nazari and M.-J. Hosseini, Int. J. Food Prop., 2016, 19, 1983-1993.

16 K. D. Bahadur and D. Anjeeta, Int. Res. J. Environ. Sci., 2014, 3, 64-69.

17 K. D. Bahadur and B. Badri, Res. J. Chem. Sci., 2014, 2231, 606X. 
18 K. Zhang, Y. Hu and G. Li, Talanta, 2013, 116, 712-718.

19 J. Chen, S. Pang, L. He and S. R. Nugen, Biosens. Bioelectron., 2016, 85, 726-733.

20 G.-P. Cao, R.-Y. Yang, Y.-F. Zhuang, D. Zuo and Y.-H. Wang, Anal. Bioanal. Chem., 2017, 409, 4637-4646.

21 A. A. Chetty and S. Prasad, Food Chem., 2016, 197, 503-508.

22 S. Wang, K. Lin, N. Chen, D. Yuan and J. Ma, Talanta, 2016, 146, 744-748.

23 H. Abdolmohammad-Zadeh and E. Rahimpour, Talanta, 2015, 144, 769-777.

24 Z. Lin, W. Xue, H. Chen and J.-M. Lin, Anal. Chem., 2011, 83, 8245-8251.

25 S. Yang and M. E. Meyerhoff, Electroanalysis, 2013, 25, 25792585.

26 J. Davis, M. J. Moorcroft, S. J. Wilkins, R. G. Compton and M. F. Cardosi, Analyst, 2000, 125, 737-742.

27 W. Liu, Z. Du, Y. Qian and F. Li, Sens. Actuators, B, 2013, 176, 927-931.

28 W. He, L. Luo, Q. Liu and Z. Chen, Anal. Chem., 2018, 90, 4770-4775.

29 I. Abdulazeez, C. Basheer and A. A. Al-Saadi, J. Mol. Liq., 2018, 264, 58-65.

30 F. Salimi, M. Kiani, C. Karami and M. A. Taher, Optik, 2018, 158, 813-825.

31 B. Kaur, N. Kaur and S. Kumar, Coord. Chem. Rev., 2018, 358, 13-69.

32 N. Adarsh, M. Shanmugasundaram and D. Ramaiah, Anal. Chem., 2013, 85, 10008-10012.

33 X. Chen, S. Yu, L. Yang, J. Wang and C. Jiang, Nanoscale, 2016, 8, 13669-13677.

34 M. H. Ibrahim, Z. Xue, M. I. Shinger, H. I. Abdu, L. Xiong, D. Shan and X. Lu, Spectrochim. Acta, Part A, 2018, 13861425.

35 Y. Wang, C. Zhang, X. Chen, B. Yang, L. Yang, C. Jiang and Z. Zhang, Nanoscale, 2016, 8, 5977-5984.

36 Z. Xue, X. Fu, H. Rao, M. H. Ibrahim, L. Xiong, X. Liu and X. Lu, Talanta, 2017, 174, 667-672.

37 J. Zhang, L. He, P. Chen, C. Tian, J. Wang, B. Liu, C. Jiang and Z. Zhang, Nanoscale, 2017, 9, 1599-1606.

38 Q.-H. Wang, L.-J. Yu, Y. Liu, L. Lin, R.-g. Lu, J.-p. Zhu, L. He and Z.-L. Lu, Talanta, 2017, 165, 709-720.

39 S. S. Ravi, L. R. Christena, N. SaiSubramanian and S. P. Anthony, Analyst, 2013, 138, 4370-4377.

40 H. Li, Z. Cui and C. Han, Sens. Actuators, B, 2009, 143, 87-92. 41 N. Nath and A. Chilkoti, Anal. Chem., 2002, 74, 504-509.

42 A. M. Idris, M. I. Shinger, S. Barkaoui, K. Khan, H. I. Abdu, M. M. Edris and X. Lu, Mater. Res. Bull., 2018, 102, 100-107. 43 V. V. Kumar and S. P. Anthony, RSC Adv., 2014, 4, 6471764724 .
44 P. Vasileva, T. Alexandrova and I. Karadjova, J. Chem., 2017, 2017, 2090-9063.

45 B. Roy, P. Bairi and A. K. Nandi, Analyst, 2011, 136, 36053607.

46 V. V. Kumar and S. P. Anthony, Sens. Actuators, B, 2014, 191, 31-36.

47 Y.-r. Ma, H.-y. Niu and Y.-q. Cai, Chem. Commun., 2011, 47, 12643-12645.

48 G. Wu, C. Dong, Y. Li, Z. Wang, Y. Gao, Z. Shen and A. Wu, RSC Adv., 2015, 5, 20595-20602.

49 U. B. Patel, V. N. Mehta, M. A. Kumar and S. K. Kailasa, Res. Chem. Intermed., 2013, 39, 771-779.

50 W. L. Daniel, M. S. Han, J.-S. Lee and C. A. Mirkin, J. Am. Chem. Soc., 2009, 131, 6362-6363.

51 N. Xiao and C. Yu, Anal. Chem., 2010, 82, 3659-3663.

52 Z. Chen, Z. Zhang, C. Qu, D. Pan and L. Chen, Analyst, 2012, 137, 5197-5200.

53 V. V. Kumar and S. P. Anthony, Anal. Chim. Acta, 2014, 842, 57-62.

54 J. A. Jacob, H. S. Mahal, N. Biswas, T. Mukherjee and S. Kapoor, Langmuir, 2008, 24, 528-533.

55 Y. Ma, H. Niu, X. Zhang and Y. Cai, Analyst, 2011, 136, 41924196.

56 Y. Shang, D. Gao, F. Wu and X. Wan, Microchim. Acta, 2013, 180, 1317-1324.

57 S. S. Gowda, S. Rajasowmiya, V. Vadivel, S. B. Devi, A. C. Jerald, S. Marimuthu and N. Devipriya, Toxicol. In Vitro, 2018, 52, 170-177.

58 P. Mulvaney, Langmuir, 1996, 12, 788-800.

59 S. Annur, S. J. Santosa, N. H. Aprilita, N. T. Phuong and N. Van Phuocs, Asian J. Microbiol. Biotechnol. Environ. Sci., 2018, 1, 76-83.

60 Y. Zhou, H. Zhao, C. Li, P. He, W. Peng, L. Yuan, L. Zeng and Y. He, Talanta, 2012, 97, 331-335.

61 Y.-F. Huang, H.-P. Zhu, G.-K. Liu, D.-Y. Wu, B. Ren and Z.-Q. Tian, J. Am. Chem. Soc., 2010, 132, 9244-9246.

62 H.-J. Jeon, S.-C. Yi and S.-G. Oh, Biomaterials, 2003, 24, 49214928.

63 C. Jayaseelan and A. A. Rahuman, Parasitol. Res., 2012, 111, 1369-1378.

64 P. Velmurugan, J. Shim, S. Kamala-Kannan, K. J. Lee, B. T. Oh, V. Balachandar and B. T. Oh, Biotechnol. Prog., 2011, 27, 273-279.

65 H. M. Ibrahim, J. Radiat. Res. Appl. Sci., 2015, 8, 265-275.

66 C.-P. Gong, S.-C. Li and R.-Y. Wang, J. Photochem. Photobiol., $B, 2018,183,137-141$.

67 M. Govarthanan, T. Selvankumar, K. Manoharan, R. Rathika, K. Shanthi, K.-J. Lee, M. Cho, S. Kamala-Kannan and B.-T. Oh, Int. J. Nanomed., 2014, 9, 1593. 